\title{
Macro and micro spectroscopy parameters of cancerous and healthy gastrointestinal tissues
}

\author{
Tsanislava Genova ${ }^{1 *}$, Ekaterina Borisova ${ }^{1,2}$, Oksana Semyachkina-Glushkovskaya ${ }^{2}$, Dmitry Gorin ${ }^{2}$, \\ Daniil Bratashov ${ }^{2}$, and Ivan Terziev ${ }^{3}$ \\ ${ }^{1}$ Institute of Electronics, Bulgarian Academy of Sciences, 72 Tsarigradsko Chaussee blvd., Sofia 1784, Bulgaria \\ ${ }^{2}$ Saratov State University, 83 Astrahanskaya str., Saratov 410012, Russian Federation \\ ${ }^{3}$ University Hospital "Tsaritsa Yoanna"-ISUL, 8 Bialo More str., Sofia 1527, Bulgaria \\ * e-mail: ts.genova@gmail.com
}

\begin{abstract}
New more sensitive diagnostic modalities, for either analysis of biopsy tissue samples or on site, in vivo microscopy tissue examination, emerge to aid the detection and accurate diagnosis of gastrointestinal cancer. One of the most intensively investigated techniques is the fluorescence spectroscopy approach.

We present our investigation of the detailed macro and micro fluorescence characteristics of ex-vivo fresh and fixed tissue samples. The fluorescence spectra of unprocessed cancerous and healthy gastrointestinal (GIT) tissue, obtained with two approaches fluorescence and synchronous fluorescence spectroscopy were compared with microspectroscopy performed with laser scanning confocal fluorescence microscopy (LSCFM) system in Lambda-scan regime over fixed tissue samples.

The evaluated optical macro- and micro- spectroscopy characteristics are presented and disscused in order to improve the current understanding of the origin of autofluorescence contrast between healthy and cancerous tissues and its application for improvement of the existing diagnostic modalities. (C) 2017 Journal of Biomedical Photonics \& Engineering.
\end{abstract}

Keywords: Autofluorescence; gastrointestinal tissues; colon carcinoma; confocal fluorescent microscopy (CFM).

Paper \#3264 received 30 Nov 2017; revised manuscript received 17 Dec 2017; accepted for publication 18 Dec 2017; published online 31 Dec 2017. doi: 10.18287/JBPE17.03.040305. [Saratov Fall Meeting 2017 Special Issue].

\section{References}

1. L. A. Torre, F. Bray, R. L. Siegel, J. Ferlay, J. Lortet-Tieulent, and A. Jemal, "Global cancer statistics, 2012," CA: A Cancer Journal for Clinicians 65(2), 87-108 (2015).

2. A. B. Benson III, "Epidemiology, Disease Progression, and Economic Burden of Colorectal Cancer," Journal of Managed Care Pharmacy 13(6), S5-S18 (2007).

3. N. Chaudhary, V. Kumaran, V. Varma, S. Kapoor, N. Mehta, and S. Nundy, "Post-Resection Surveillance in GI Cancers," The Indian Journal of Surgery 76(5), 382-391 (2014).

4. J. J. Telford, and R. A. Enns, "Endoscopic missed rates of upper gastrointestinal cancers: parallels with colonoscopy," The American Journal of Gastroenterology 105, 1298-1300 (2010).

5. M. Song, and T. Ang, "Early detection of early gastric cancer using image-enhanced endoscopy: Current trends," Gastrointestinal Intervention 3(1), 1-7 (2014).

6. M. Fujiya, and Y. Kohgo, "Image-enhanced endoscopy for the diagnosis of colon neoplasms," Gastrointestinal Endoscopy 77(1), 111-118.e5 (2013).

7. A. Johansson, K. Kromer, R. Sroka, and H. Stepp, "Clinical optical diagnostics: Status and perspectives," Medical Laser Application 23(4), 155-174 (2008).

8. M. Hasan, and M. Wallace, "Image-enhanced endoscopy," Clinical Update 16(4), 1-5 (2009). 
9. V. Subramanian, and K. Ragunath, “Advanced Endoscopic Imaging: A Review of Commercially Available Technologies," Clinical Gastroenterology and Hepatology 12(3), 368-376.e1 (2014).

10. D. Roblyer, R. Richards-Kortum, K. Sokolov, A. K. El-Naggar, M. D. Williams, C. Kurachi, and A. M. Gillenwater, "Multispectral optical imaging device for in vivo detection of oral neoplasia," Journal of Biomedical Optics 13(2), 024019 (2008).

11. D. Pantalone, F. Andreoli, F. Fusi, V. Basile, G. Romano, G. Giustozzi, L. Rigacci, R. Alterini, and M. Monici, "Multispectral Imaging Autofluorescence Microscopy in Colonic and Gastric Cancer Metastatic Lymph Nodes," Clinical Gastroenterology and Hepatology 5(2), 230-236 (2007).

12. D. Pantalone, M. Monici, G. Romano, F. Cialdai, R. Santi, F. Fusi, C. Comin, and P. Bechi, "Colonic and gastric cancer metastatic lymph nodes: applications of autofluorescence-based techniques," Oncology Reviews 4(1), 15-23 (2010).

13. R. Richards-Kortum, R. P. Rava, R. E. Petras, M. Fitzmaurice, M. Sivak, and M. S. Feld, "Spectroscopic diagnosis of colonic dysplasia," Photochemistry and Photobiology 53(6), 777-786 (1991).

14. G. Bottiroli, A. C. Croce, D. Locatelli, R. Marchesini, E. Pignoli, S. Tomatis, C. Cuzzoni, S. Di Palma, M. Dalfante, and P. Spinelli, "Natural fluorescence of normal and neoplastic human colon: a comprehensive "ex vivo" study," Lasers in Surgery and Medicine 16(1), 48-60 (1995).

15. H. Zheng, A. Weiss, R. Cline, and C MacAulay, "Real-time endoscopic fluorescence imaging for early cancer detection in the gastrointestinal tract," Bioimaging 6, 151-165 (1998).

16. B. H. Li, and S. S. Xie, "Autofluorescence excitation-emission matrices for diagnosis of colonic cancer," World Journal of Gastroenterology 11(25), 3931-3934 (2005).

17. B. H. Li, S. S. Xie, and Z. Zhang, "Steady-State and Time-Resolved Fluorescence Spectra for Detection of Colonic Cancer," Proceedings of SPIE 5967, 59670A (2006).

18. X. Shao, W. Zheng, and Z. Huang, "In vivo diagnosis of colonic precancer and cancer using near-infrared autofluorescence spectroscopy and biochemical modeling," Journal of Biomedical Optics 16(6), 067005 (2011).

19. A. Croce, and G. Bottiroli, “Autofluorescence Spectroscopy and Imaging: A Tool for Biomedical Research and Diagnosis," European Journal of Histochemistry 58(4), 2461 (2014).

20. Y. DeClerck, "Interactions between tumour cells and stromal cells and proteolytic modification of the extracellular matrix by metalloproteinases in cancer," European Journal of Cancer 36(10), 1258-1268 (2000).

21. K. T. Moesta, B. Ebert, T. Handke, D. Nolte, C. Nowak, W. E. Haensch, R. K. Pandey, T. J. Dougherty, H. Rinneberg, and P. M. Schlag, "Protoporphyrin IX occurs naturally in colorectal cancers and their metastases," Cancer Research 61(3), 991-999 (2001).

22. F. Broek, P. Fockens, and E. Dekker, "Review article: new developments in colonic imaging," Alimentary Pharmacology \& Therapeutics 26, 91-99 (2007).

23. H. Aihara, K. Sumiyama, S. Saito, H. Tajiri, and M. Ikegami, "Numerical analysis of the autofluorescence intensity of neoplastic and non-neoplastic colorectal lesions by using a novel videoendoscopy system," Gastrointestinal Endoscopy 69, 726-733 (2009).

24. T. Genova, E. Borisova, N. Penkov, B. Vladimirov, A. Zhelyazkova, and L. Avramov, "Excitation-emission matrices and synchronous fluorescence spectroscopy for the diagnosis of gastrointestinal cancers," Quantum Electronics 46(6), 510 (2016).

25. E. Borisova, L. Plamenova, M. Keremedchiev, B. Vladimirov, and L. Avramov, "Endogenous and exogenous fluorescence spectroscopy of gastrointestinal tumours - in vitro studies", Journal of Optoelectronics and Advanced Materials 16(9), 1196-1205 (2014).

26. T. Genova, E. Borisova, Al. Zhelyazkova, N. Penkov, B. Vladimirov, I. Terziev, O. SemyachkinaGlushkovskaya, and L. Avramov, "Colorectal cancer stage evaluation using synchronous fluorescence spectroscopy technique," Optical and Quantum Electronics 48(8), 1-10 (2016).

27. L. Avramov, E. Borisova, Ts. Genova, Al. Zhelyazkova, L. Angelova, M. Keremedchiev, N. Penkov, I. Terziev, B. Vladimirov, and O. Semyachkina-Glushkovskaya, "Synchronous autofluorescence spectroscopy of gastrointestinal tumours - tool for endogenous fluorophores evaluation," Optoelectronics and Advanced Materials - Rapid Communications 9(9-10), 1234-1238 (2015).

28. T. Vo-Dinh, "Principle of Synchronous Luminescence (SL) technique for biomedical diagnostics," Proceedings of SPIE 3911, 42-49 (2000).

29. S. Kakar, C. Shi, M. Berho, D. K. Driman, P. Fitzgibbons, W. L. Frankel, K. A. Hill, J. Jessup, A. M. Krasinskas, and M. K. Washington, "Protocol for the Examination of Specimens From Patients With Primary Carcinoma of the Colon and Rectumm," College of American Pathologists (CAP) (2017).

30. M. S. Viegas, T. C. Martins, F. Seco, and A. do Carmo, "An improved and cost-effective methodology for the reduction of autofluorescence in direct immunofluorescence studies on formalin-fixed paraffin-embedded tissues," European Journal of Histochemistry 51(1), 59-66 (2007). 
31. J. Aisa, M. Lahoz, P. Serrano, M. C. Pérez-Castejón, C. Junquera, M. C. Martínez-Ciriano, N. Pes, and A. Vera-Gil, "Acetylcholinesterase positive and paraformaldehyde induced fluorescence positive innervation in the upper eyelid of the sheep (Ovis aries)," Histology and Histopathology 16, 487-496 (2001).

32. N. D. Kirkpatrick, M. A. Brewer, and U. Utzinger, "Endogenous optical biomarkers of ovarian cancer evaluated with multiphoton microscopy," Cancer Epidemiology Biomarkers \& Prevention 16(10), 2048-57 (2007).

33. M. W. Conklin, P. P. Provenzano, K. W. Eliceiri, R. Sullivan, and P. J. Keely, "Fluorescence lifetime imaging of endogenous fluorophores in histopathology sections reveals differences between normal and tumor epithelium in carcinoma in situ of the breast," Cell Biochemistry and Biophysics 53(3), 145-157 (2009).

34. R. M. Williams, A. Flesken-Nikitin, L. H. Ellenson, D. C. Connolly, T. C. Hamilton, A. Y. Nikitin, and W. R. Zipfel, "Strategies for High-Resolution Imaging of Epithelial Ovarian Cancer by Laparoscopic Nonlinear Microscopy," Translational Oncology 3(3), 181-194 (2010).

35. J. Vivian, and P. Callis, "Mechanisms of Tryptophan Fluorescence Shifts in Proteins," Biophysical Journal 80(5), 2093-2109 (2001).

36. P. Callis, and B. Burgess, "Tryptophan fluorescence shifts in proteins from hybrid simulations: an electrostatic approach," The Journal of Physical Chemistry B 101(46), 9429-9432 (1997).

37. G. Palmer, C. Zhu, T.M. Breslin, F. Xu, K.W. Gilchrist, and N. Ramanujam, "Comparison of multiexcitation fluorescence and diffuse reflectance spectroscopy for the diagnosis of breast cancer," IEEE Transactions on Biomedical Engineering 50(11), 1233-1242 (2003).

38. Y. Li, Separation of endogenous fluorophores in normal and cancer cells, $\mathrm{PhD}$ thesis, University of Iowa, 2009.

\section{Introduction}

The wide spread of oncologic diseases in gastrointestinal tract (GIT) and high mortality among the patients [1], entitle its early diagnosis and effective treatment as a primary public health concern.

The standard procedure for cancer detection in GIT includes endoscopic surveillance and biopsy. Their methodical application proves to be effective in reducing of mortality rates and improvement of patient's quality of life [2, 3]. However the rigorous biopsy protocols are costly and time consuming. The accuracy of the current diagnostic procedure relies entirely on the physicians' experience and it is limited by the high probability of miss rates [4]. Therefore new sensitive diagnostic modalities, for either analysis of biopsy tissue samples or on site, in vivo tissue diagnostic evaluation, are necessary. One of the most intensively investigated techniques, for overcoming the limitation of the standard diagnostic procedure, are the fluorescence spectroscopy and multispectral imaging approaches [4-12].

Recent advances in the understanding of the process of cancerogenesis and its footprints in tissue autofluorescence spectra allow the application of autofluorescence contrast in "red-flag" techniques for clinical diagnostics $[5,6,7]$.

At general clinical application of autofluorescence contrast in GIT cancer diagnosis is based on the macroscopic manifestation of the difference in the autofluorescence of cancerous and healthy tissue, predominantly green fluorescence $(450-550 \mathrm{~nm})$ of healthy mucosa and red fluorescence $(600-700 \mathrm{~nm})$ of hyperplasic mucosa. Most often the diagnostic parameter is considered the ratio between the intensities of autofluorescence in the two regions, with different systems using different spectral ranges within the green and red regions $[6,8,9]$. The origin of the difference in autofluorescence of cancerous and healthy GIT tissues is attributed to few main endogenous fluorophores that are affected by the process of oncogenesis: amino acids - tyrosine and tryptophan, co-enzymes nicotinamide adenine dinucleotide $(\mathrm{NAD}(\mathrm{P}) \mathrm{H})$ and flavine adenine dinucleotide (FAD), structural proteins collagen and elastin, as well as their cross-links, and porphyrins. In ex vivo studies of autofluorescence of normal and cancerous tissues with colorectal origin, reduced intensity of $\mathrm{NAD}(\mathrm{P}) \mathrm{H}$ 's fluorescence maxima have been repeatedly observed [13-18]. NAD $(\mathrm{P}) \mathrm{H}$ is fluorescent only in its reduced form, its fluorescence depends on the quantity, redox and free-bound state. Hypoxia in cancerous tissues could increase the fluorescence of $\mathrm{NAD}(\mathrm{P}) \mathrm{H}$ by ceasing its oxidation, however it is typical mostly for solid tumours. It was demonstrated that there are numerous metabolic pathways affecting the redox stated of $\mathrm{NAD}(\mathrm{P}) \mathrm{H}$ and also that an alteration in bound to free $\mathrm{NAD}(\mathrm{P}) \mathrm{H}$ ratio could cause decrease in NAD(P)H's fluorescence and also red shift in its maximum [19]. The intensity of the fluorescence of collagen is reduced due to disruption of intra and extracellular matrices, caused by the poor differentiation and excessive proliferation of cancerous cells [20]. Excessive amount of porphyrins is found in cancerous cells, as a result of bypassing effect of the inhibited activity of the enzyme ferrohelatases in cancerous cells [21]. It is considered that the combination between reduced fluorescence from collagen $(400 \mathrm{~nm}, 405 \mathrm{~nm})$ and increased fluorescence of porphyrins $(630 \mathrm{~nm}, 690 \mathrm{~nm})$ is the reason for the characteristic red fluorescence of cancerous tissues.

However, the current application of autofluorescence based diagnostic techniques is limited mostly by insufficient specificity and misjudging 
between cancer and inflammation, due to higher porphyrins content characteristic for both pathologies $[5,22,23]$.

The more efficient and robust application of autofluorescence based endoscopic techniques for detection of GIT cancer requires extensive and meticulous evaluation of autofluorescence spectral peculiarities characteristic for cancerous tissue fluorescence, their origin, significance and specificity.

In our previous works we have presented detailed investigations over the main fluorescence characteristics of gastrointestinal tissues, specific fluorophores and their significance for differentiation between cancerous and healthy GIT tissues [24-27]. We have also demonstrated implementation of synchronous fluorescence spectroscopy (SFS) [28] for tissue investigation and its superior to standard fluorescence spectroscopy, diagnostic potential [25-27].

Hereby we present our investigation and comparison of the macro and micro fluorescence characteristics of ex-vivo fresh and fixed tissue samples, through fluorescence and synchronous fluorescence spectroscopy, and laser scanning confocal fluorescence microscopy respectively. As a part of our work towards better understanding of the underlying autofluorescence contrast between cancerous and healthy gastrointestinal tissue and its application for cancer detection and diagnostics.

\section{Materials and Methods}

\subsection{Unprocessed tissue samples}

Investigated biological tissue samples originate from gastrointestinal tract, colon and rectum. The number of samples collected is as follows: eighteen colon adenocarcinomas, two tubulovillous adenomas (polyp) and four adenocarcinomas of rectum. The tissue samples are excised during standard surgical procedure for tumour removal and consist of tumour and surrounding healthy tissue - safety region, excised according protocols [29] and stored in safe-keeping modified Krebs solution $(\mathrm{NaCl}, \mathrm{KCl}$, glucose, taurine, hepes, pyruvic acid, calcium chloride dehydrate) and isothermal conditions prior the spectral measurements.

\subsection{Processed tissue samples}

Investigated fixed tissue samples are unstained, deparaffinized and rehydrated histology tissue slides. Two consecutive slides were cut for every sample. After cutting the tissue slides and positioning on the glass slides (Leica X-tra Adhesive) all slides were deparaffinized by xylene (soak for $5 \mathrm{~min}$.).

According to the histopathology diagnosis, samples included in this study are five samples of colon adenocarcinoma, two samples of tubulovillous adenoma and two samples of adenocarcinoma of rectum.

All tissue samples were obtained with approval by Ethics committee of University Hospital "Tsaritsa Yoanna - ISUL" in the frames of collaboration between the hospital and Institute of Electronics, Bulgarian Academy of Sciences.

\subsection{Fluorescence spectroscopy}

The fluorescence spectroscopy measurements were obtained using spectrofluorimeter FluoroLog 3 (HORIBA Jobin Yvon, France). For fluorescence spectroscopy the applied excitation wavelength is 405 $\mathrm{nm}$, and emission was detected in the range of 415-800 $\mathrm{nm}$. The SFS method is characterized with simultaneous scanning of both the excitation and emission wavelengths, while a constant wavelength interval (offset) is kept between them. For SFS excitation used was in the range of $280-440 \mathrm{~nm}$, with a step of $1 \mathrm{~nm}$ and $60 \mathrm{~nm}$ and $90 \mathrm{~nm}$ offset. For each sample the normal and cancerous areas were measured spectroscopically.

\subsection{Fluorescence Microspectroscopy}

Imaging and microspectroscopy was performed with confocal laser scanning microscopy system Leica TCS SP (Leica Inc.), applying excitation at $405 \mathrm{~nm}$ excitation. Images were acquired using a 40x magnification objective with a numerical aperture of 0.75 . The field of view for each image was $775 \times 775$ $\mu \mathrm{m}$. In Lambda-scan ${ }^{\circledR}$ regime of the microscope the fluorescence spectra of histology tissue slides were evaluated over series of images throughout the slide thickness, which is $12 \mu \mathrm{m}$ for healthy area and area with distinctive cancer alterations in the tissue pattern. The spectral and image detection eas the range of 420-680 nm.

\section{Results and Discussion}

Fig. 1(a) represents the obtained LSCFM image of colon adenocarcinoma, where the typical for oncogenesis distortion of the shape of crypts and their density is higher than normal with almost missing the natural lamina propria, fluorescence spectra of this area named "cancerous" and fluorescence spectra of healthy area on the sample (Fig. 1(b)).

Fig. 2(a) represents the obtained LSCFM image of tubulovillous adenoma (polyp), where the typical healthy crypts, "border" area and area with complete loss of differentiation is observed. Figure 3(a) represents the obtained LSCFM image of adenocarcinoma of rectum, where mostly complete loss of differentiation is observed.

For the fluorescence spectra of the fixed tissue sections, a slight red-shift for the autofluorescence of the cancerous areas is observed (Fig. 1(b); Fig. 2(b); Fig. 3(b)). Since this red-shift is very slight, boxwhiskers plot with intensity values for fluorescence at $600 \mathrm{~nm}$ for five cancerous and healthy parts of the included samples with the respective diagnosis are presented (Fig. 1(c); Fig. 2(c); Fig. 3(c)). For every plot the whiskers mark the standard deviation with 1.5 coefficient; upper and lower box ranges reflect the $25 / 75$ th percentiles, respectively; the mean value is 


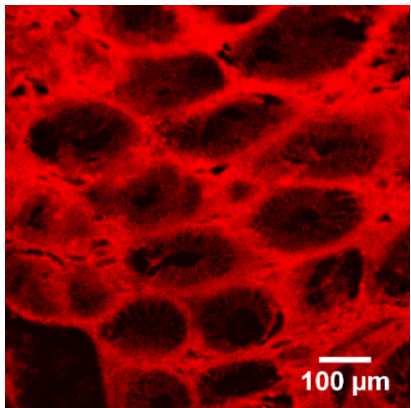

(a)

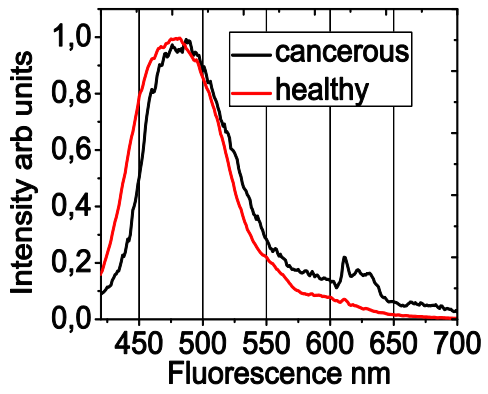

(d)

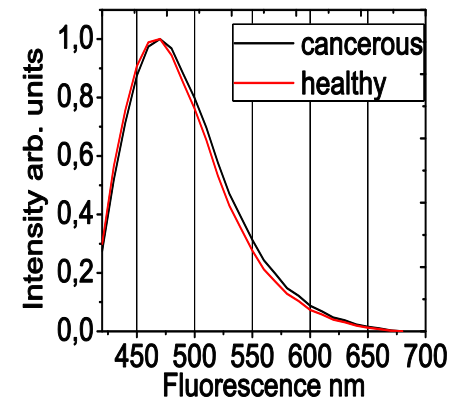

(b)

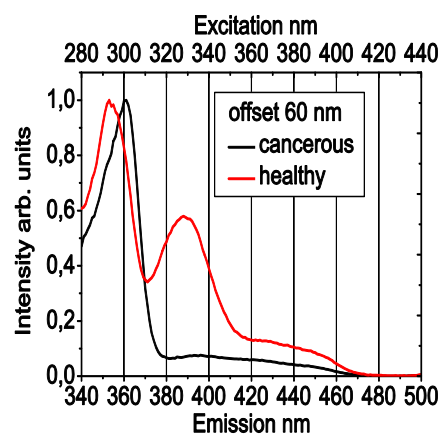

(e)

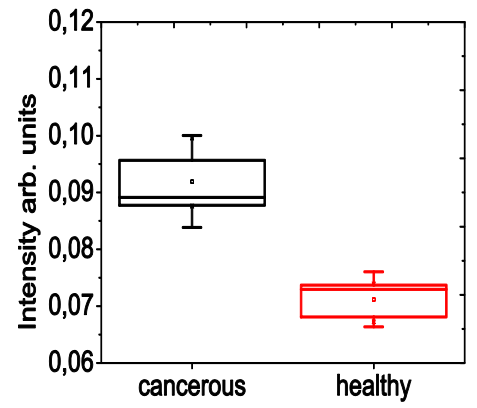

(c)

Excitation $\mathrm{nm}$ 280300320340360380400420440

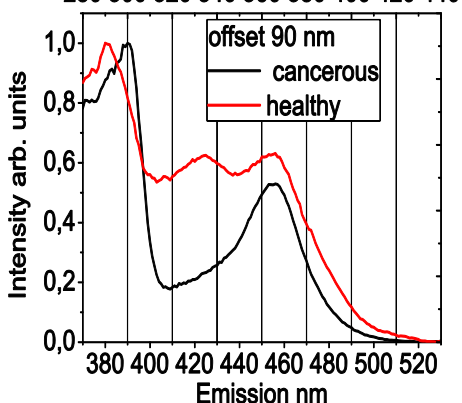

(f)

Fig. 1 Obtained macro and micro data for colon adenocarcinoma. LSCFM image of fixed tissue section for excitation of $405 \mathrm{~nm}$ (a); fluorescence spectra of fixed tissue section for excitation of $405 \mathrm{~nm}$ (b); intensity values at $600 \mathrm{~nm}$ for 5 cancerous and healthy parts of the fixed tissue sample (c); fluorescence spectra of unprocessed tissue sample of colon adenocarcinoma for excitation of $405 \mathrm{~nm}$ (d); SFS spectra of unprocessed tissue sample of colon adenocarcinoma for offset of $60 \mathrm{~nm}$ (e) and $90 \mathrm{~nm}$ (f). All fluorescence spectra are from single sample and normalized according to their maximum.

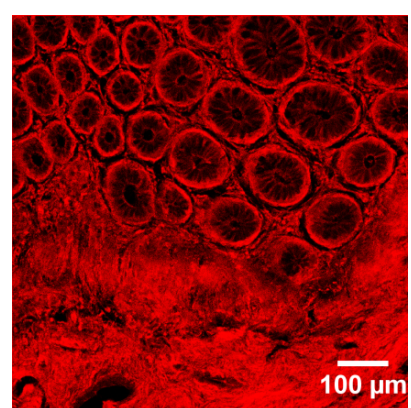

(a)

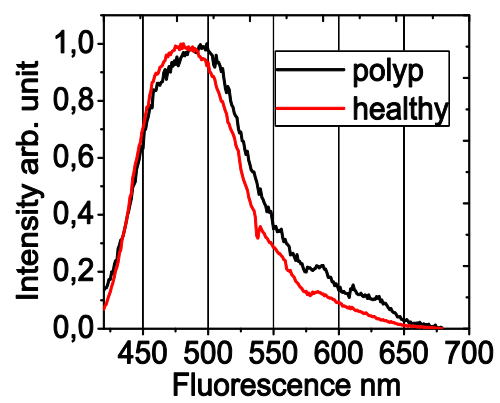

(d)

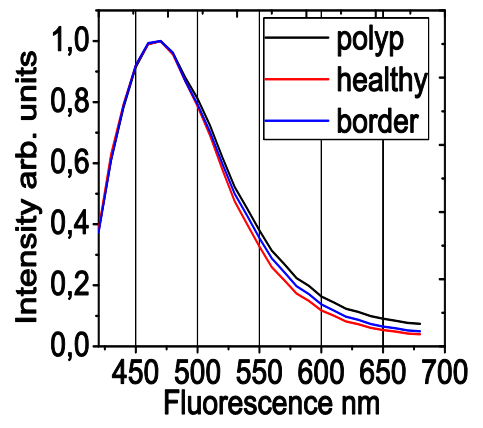

(b)

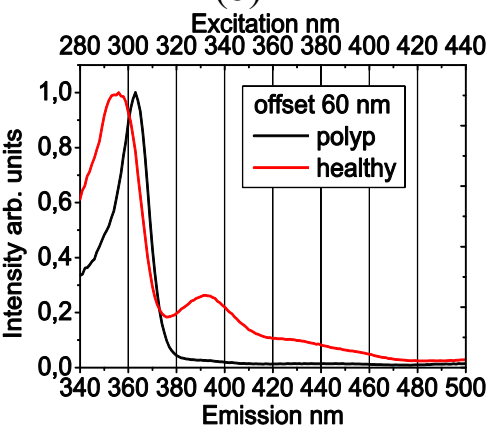

(e)

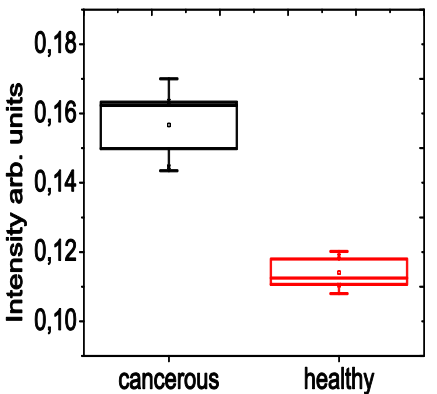

(c)

Excitation $\mathrm{nm}$ 280300320340360380400420440

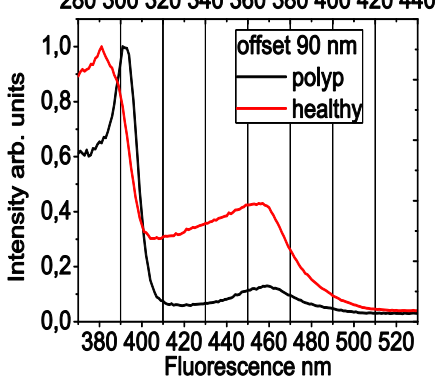

(f)

Fig. 2 Obtained macro and micro data for tubulovillous adenoma. LSCFM image of fixed tissue section for excitation of $405 \mathrm{~nm}$ (a); fluorescence spectra of fixed tissue section for excitation of $405 \mathrm{~nm}$ (b); intensity values at $600 \mathrm{~nm}$ for 5 cancerous and healthy parts of the fixed tissue sample (c); fluorescence spectra of unprocessed tissue sample of colon adenoma for excitation of $405 \mathrm{~nm}$ (d); SFS spectra of unprocessed tissue sample of colon adenoma for offset of $60 \mathrm{~nm}$ (e) and $90 \mathrm{~nm}$ (f). All fluorescence spectra are from single sample and normalized according to their maximum. 


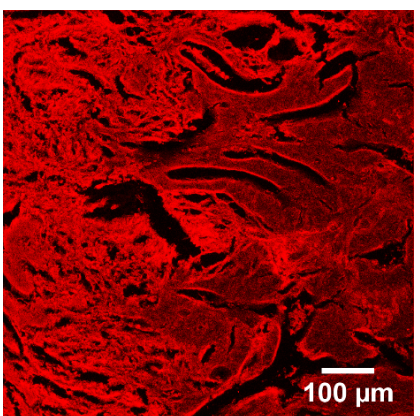

(a)

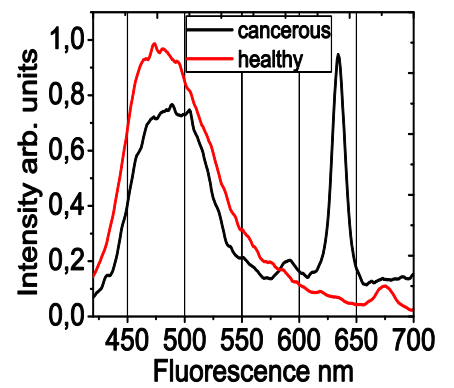

(d)

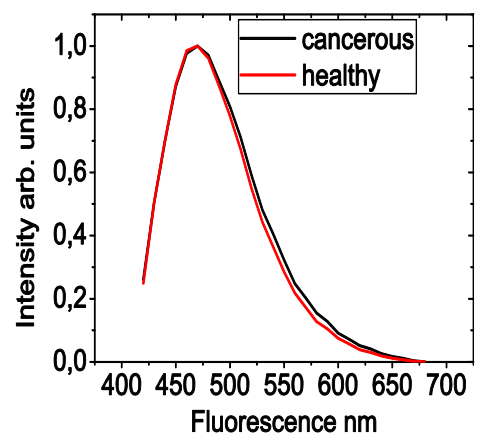

(b)

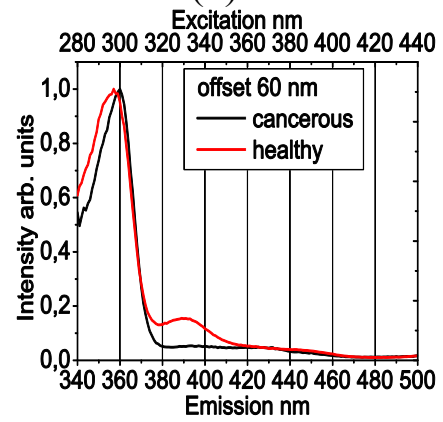

(e)

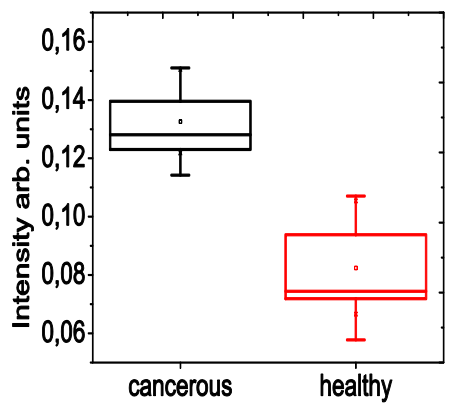

(c)

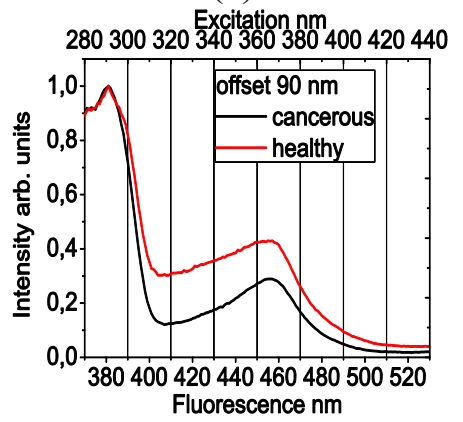

(f)

Fig. 3 Obtained macro and micro data for adenocarcinoma of rectum. LSCFM image of fixed tissue section for excitation of $405 \mathrm{~nm}$ (a); fluorescence spectra of fixed tissue section for excitation of $405 \mathrm{~nm}$ (b); intensity values at $600 \mathrm{~nm}$ for 5 cancerous and healthy parts of the fixed tissue sample (c); fluorescence spectra of unprocessed tissue sample of colon adenocarcinoma for excitation of $405 \mathrm{~nm}$ (d); SFS spectra of unprocessed tissue sample of colon adenocarcinoma for offset of $60 \mathrm{~nm}$ (e) and $90 \mathrm{~nm}$ (f). All fluorescence spectra from single sample and are normalized according to their maximum.

represented with line across the box and the median value is represented with square. This representation of the data demonstrates that the observed red-shift of the fluorescence of cancerous tissues in fixed tissue samples is statistically significant. The detected fluorescence could be contributed to NAD(P)H, FAD, porphyrins and mostly the structural proteins collagen and elastin, since the applied excitation of $405 \mathrm{~nm}$ is adequate for their excitation, the other fluorophores are more affected by the tissue processing, also some of the fluorescence signal could be attributed to formalin fixation [30, 31]. The most discussed causes of the observed spectral shift towards longer wavelengths are an increase in the $\mathrm{FAD} / \mathrm{NAD}(\mathrm{P}) \mathrm{H}$ ratio [32], alteration in the $\mathrm{NAD}(\mathrm{P}) \mathrm{H}_{\text {bound/free }}$ ratio $[33,34]$ and the fluorescence of porphyrins [34, 35].

The fluorescence spectra of unprocessed tissue samples after excitation at $405 \mathrm{~nm}$ (Fig. 1(d); Fig. 2(d); Fig. 3(d)) exhibits one main fluorescence maxima due to the fluorescence of $\mathrm{NAD}(\mathrm{P}) \mathrm{H}$ and structural proteins and for the samples of colon adenocarcinoma and adenocarcinoma of the rectum (Fig. 1(d); Fig. 3(d)) characteristic fluorescence maxima of porphyrins around $630 \mathrm{~nm}$ that is typical for higher grade dysplasia.

Synchronous fluorescence spectra for excitation 280-440 nm and offset $60 \mathrm{~nm}$ (Fig. 1(e); Fig. 2(e); Fig. $3(\mathrm{e})$ ) consists of two maxima for the healthy tissue fluorescence contributed to amino acids tyrosine and tryptophan and collagen and only the maxima of the amino acids for cancerous tissue. The red-shift in the fluorescence maxima of the amino acids is consistent and prominent feature for the presented SFS spectra of cancerous tissues. The reason for this spectral shift could be the high sensitivity of tryptophan's fluorescence to the environment such as $\mathrm{pH}$ and local electric field [19, 34, 36] Red-shift was also observed for the fluorescence maxima of tryptophan in breast cancer tissue's fluorescence [37]. The lower fluorescence intensity of collagen for cancerous tissues is its typical spectral hallmark [24, 26, 27, 28] and in the presented results it is negligible low in comparison with collagen fluorescence maxima in healthy tissue spectra.

Synchronous fluorescence spectra for excitation $280-440 \mathrm{~nm}$ and offset $90 \mathrm{~nm}$ (Fig. 1(f); Fig. 2(f); Fig. 3(f)) have two prominent fluorescence maxima - first one is contributed to tryptophan and second one is $\mathrm{NAD}(\mathrm{P}) \mathrm{H}$ fluorescence maxima. Except for the colon adenocarcinoma (Fig. 1(f)) where and an additional maximum around $420 \mathrm{~nm}$ for healthy tissue fluorescence is distinguishable and could be attributed to collagen and its cross-links. For the maxima of $\mathrm{NAD}(\mathrm{P}) \mathrm{H}$ there is no prominent red spectral shift, the only difference between the spectra of cancerous and healthy fluorescence for this maxima is in the intensity, which is lower for cancerous tissues fluorescence and it was already observed [11, 28, 38]. 


\section{Conclusions}

Early detection of cancer plays an important role in global cancer control initiatives as it significantly increases the survival rate and improves quality of life of the patients. The fluorescence spectroscopy and/or imaging has already been proven to increase the image contrast and detect the subtle differences between healthy epithelial tissues and early precancerous changes of GI epithelium, that would remain invisible using conventional methods of diagnostics.

Fluorescence spectroscopy and microscopy approaches provide detailed information about fluorescence spectra peculiarities that differ cancerous and healthy tissues. Nevertheless for their application in clinical practice only the most distinctive and specific optical markers should be determined, so unnecessary data processing would be eliminated. Additionally the specificity of the observed red shift in cancerous tissues fluorescence and its advantages as diagnostic parameter should be determined.

In combination with other optical markers it could be applied through both techniques fluorescence spectroscopy and microscopy, allowing a development of simplified systems, which could be used in clinical observations in vivo, or for retrospective and prospective analysis of histological slices and tissues ex vivo, as well for monitoring during surgical procedures for tumour removal.

\section{Disclosures}

The authors declare that there are no conflicts of interest related to this article.

\section{Acknowledgements}

This work was carried out in the frames and under the funding of project DNTS/Russia 01/7-23/06/2017 "Development of new optical technologies for improvement of diagnostics quality of gastric cancer", BNSF "Competitions for Financial Support for Bilateral Projects-2016-Bulgaria-Russia”. 